\title{
Predicting Dark Energy Survey Results Using the Flat Space Cosmology Model
}

\author{
Eugene Terry Tatum ${ }^{1}$, U. V. S. Seshavatharam ${ }^{2}$ \\ ${ }^{1}$ Bowling Green, KY, USA \\ ${ }^{2}$ Honorary Faculty, I-SERVE, Hyderabad, India \\ Email: ett@twc.com, seshavatharam.uvs@gmail.com
}

How to cite this paper: Tatum, E.T. and Seshavatharam, U.V.S. (2019) Predicting Dark Energy Survey Results Using the Flat Space Cosmology Model. Journal of Modern Physics, 10, 1083-1089. https://doi.org/10.4236/jmp.2019.109070

Received: July 12, 2019

Accepted: July 30, 2019

Published: August 2, 2019

Copyright $\odot 2019$ by author(s) and Scientific Research Publishing Inc. This work is licensed under the Creative Commons Attribution International License (CC BY 4.0).

http://creativecommons.org/licenses/by/4.0/

cc) (i) Open Access

\begin{abstract}
The Flat Space Cosmology (FSC) model is utilized to show how this model predicts the value of the Hubble parameter at each epoch of cosmic expansion. Specific attention in this paper is given to correlating the observable galactic redshifts since the beginning of the "cosmic dawn" reionization epoch. A graph of the log of the Hubble parameter as a function of redshift $z$ is presented as the FSC prediction of the pending Dark Energy Survey results. In the process, it is discovered that the obvious tension between the SHOES local Hubble constant value and the 2018 Planck Survey and the 2018 Dark Energy Survey global Hubble constant values may be explained by a time-variable, scalar, Hubble parameter acting in accordance with the FSC model.
\end{abstract}

\section{Keywords}

Flat Space Cosmology, Dark Energy, Hubble Parameter, SHOES Hubble Constant, $\Lambda$ CDM Concordance Model, Cosmology Theory, Early Universe

\section{Introduction and Background}

The SHOES (Supernovae, $H_{0}$, for the Equation of State of dark energy) report [1] of a "best estimate" local Hubble constant value of $73.24 \pm 1.74 \mathrm{~km} \cdot \mathrm{s}^{-1} \cdot \mathrm{Mpc}^{-1}$ is in tension with the global Hubble constant estimates of $67.36 \pm 0.54 \mathrm{~km} \cdot \mathrm{s}^{-1} \cdot \mathrm{Mpc}^{-1}$ and $67.77 \pm 1.30 \mathrm{~km} \cdot \mathrm{s}^{-1} \cdot \mathrm{Mpc}^{-1}$ reported by the 2018 Planck Survey [2] and 2018 Dark Energy Survey (DES) [3] collaborations, respectively. Given these dramat-

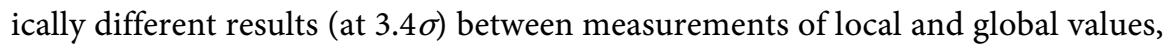
one wonders if what most cosmologists prefer to call the Hubble constant is actually a time-variable, scalar, Hubble parameter. In the next few years, this question is likely to be resolved by earth and space-based telescopes and wide-field cameras in conjunction with $3 \mathrm{D}$ computer simulations of the time evolution of 
our expanding universe. Ideally, we hope to learn the value of the Hubble parameter at each point in cosmic time beginning with the "cosmic dawn" epoch.

At present, the $\Lambda \mathrm{CDM}$ concordance model of cosmology is only seriously challenged by one other type of model, the $R_{h}=c t$ model. This type of model was introduced by Fulvio Melia in 2012 [4] and several similar models of this type soon followed. Perhaps the most successful of these models, in terms of observational correlations and predictions, is Flat Space Cosmology (FSC) [5] [6] [7] [8] [9]. The interested reader should start with a recent FSC summary paper [10], which shows how its predictive capacity and falsifiability make it preferable in many ways to the $\Lambda \mathrm{CDM}$ concordance model. To date, none of the many global predictions of the time-varying FSC model parameters (Hubble parameter, radius, mass, energy, entropy, average temperature, temperature anisotropy, etc.) have been falsified. One of the important ways in which the FSC model is superior to the $\Lambda \mathrm{CDM}$ concordance model is in its predictive capacity with respect to calculating the value of the Hubble parameter at every second of cosmic evolution from the Planck-scale epoch. FSC since its inception in 2015, for instance, has predicted a current global Hubble parameter value of 66.893 $\mathrm{km} \cdot \mathrm{s}^{-1} \cdot \mathrm{Mpc}^{-1}$, which fits the measured 2018 Planck and $2018 \mathrm{DES}$ values given above. This FSC value also fits the $66.93 \pm 0.62 \mathrm{~km} \cdot \mathrm{s}^{-1} \cdot \mathrm{Mpc}^{-1}$ value predicted by $\Lambda \mathrm{CDM}$ with 3 neutrino flavors having a mass of $0.06 \mathrm{eV}$.

It is the purpose of this paper to show how the FSC model, since its inception, has provided a means to calculate the Hubble parameter values correlating with every cosmic redshift epoch since "cosmic dawn".

\section{Results}

The relevant FSC equations useful for these calculations include:

$$
\begin{gathered}
Z \cong\left(\frac{T_{t}^{2}}{T_{o}^{2}}-1\right)^{1 / 2} \\
T_{t}^{2} R_{t} \cong 1.027246639815497 \times 10^{27} \mathrm{~K}^{2} \cdot \mathrm{m}
\end{gathered}
$$

It is trivial to show how Equations (1) and (2), in conjunction with the FSC Hubble parameter definition $\left(H_{t}=c R_{t}\right)$, can be rearranged and undergo substitution to give:

$$
T_{0}^{2}\left(z^{2}+1\right) \cong H_{t}\left[\frac{1.027246639815497 \times 10^{27} \mathrm{~K}^{2} \cdot \mathrm{m}}{c}\right]
$$

For ease of comparison, the value of the time-dependent Hubble parameter $H_{t}$ in reciprocal seconds $\left(\mathrm{s}^{-1}\right)$ can be multiplied by $3.08567758 \times 10^{19} \mathrm{~km} \cdot \mathrm{Mpc}^{-1}$ to convert $H_{t}$ to its conventional $\mathrm{km} \cdot \mathrm{s}^{-1} \cdot \mathrm{Mpc}^{-1}$ units. To accomplish this, the left-hand term in (3) is multiplied by this factor. One can now use the following equation to compare redshift $z$ values with $H_{t}$ values in $\mathrm{km} \cdot \mathrm{s}^{-1} \cdot \mathrm{Mpc}^{-1}$ :

$$
\begin{aligned}
& T_{0}^{2}\left(\mathrm{z}^{2}+1\right)\left(3.08567758 \times 10^{19} \mathrm{~km} \cdot \mathrm{Mpc}^{-1}\right) \\
& \cong H_{t}\left[\frac{1.027246639815497 \times 10^{27} \mathrm{~K}^{2} \cdot \mathrm{m}}{c}\right]
\end{aligned}
$$


Taking the square root of both sides of (4) gives:

$$
\left(z^{2}+1\right)^{1 / 2} \cong\left[H_{t}\right]^{1 / 2}\left[\frac{1.027246639815497 \times 10^{27} \mathrm{~K}^{2} \cdot \mathrm{m}}{c T_{0}^{2}\left(3.08567758 \times 10^{19} \mathrm{~km} \cdot \mathrm{Mpc}^{-1}\right)}\right]^{1 / 2}
$$

Using the $z+1$ convention for redshift, this approximates to:

$$
z+1 \cong 0.122 \sqrt{H_{t}}
$$

One can then use the knowledge that today's FSC Hubble parameter value of $66.893 \mathrm{~km} \cdot \mathrm{s}^{-1} \cdot \mathrm{Mpc}^{-1}$ corresponds to 14.617 billion years of current cosmic age in order to calculate Hubble parameters at every billion-year interval of cosmic age. The following equation is useful in this regard:

$$
H_{t} \cong H_{0}\left(\frac{14.617}{t}\right)
$$

where the $t$ value is simply the integer, or fractional number, of billions of years of cosmic age, and $H_{0}=66.893 \mathrm{~km} \cdot \mathrm{s}^{-1} \cdot \mathrm{Mpc}^{-1}$.

One can then construct the following table (Table 1) of FSC Hubble parameter values corresponding to redshift $z$ values varying from $z=0$ (corresponding to today's observed global Hubble parameter value) to $z=11.09$ (for the epoch

\begin{tabular}{|c|c|c|c|}
\hline Cosmic Age (Gyrs) & Redshift $z$ & $H_{t}\left(\mathrm{~km} \cdot \mathrm{s}^{-1} \cdot \mathrm{Mpc}^{-1}\right)$ & $\log _{10}\left(H_{t}\right)$ \\
\hline 14.617 & 0.00 & 66.893 & 1.83 \\
\hline 14 & 0.02 & 69.84 & 1.84 \\
\hline 13 & 0.06 & 75.21 & 1.88 \\
\hline 12 & 0.10 & 81.48 & 1.91 \\
\hline 11 & 0.15 & 88.89 & 1.95 \\
\hline 10 & 0.21 & 97.78 & 1.99 \\
\hline 9 & 0.27 & 108.64 & 2.04 \\
\hline 8 & 0.35 & 122.22 & 2.09 \\
\hline 7 & 0.44 & 139.68 & 2.15 \\
\hline 6 & 0.56 & 162.96 & 2.21 \\
\hline 5 & 0.71 & 195.55 & 2.29 \\
\hline 4 & 0.91 & 244.44 & 2.39 \\
\hline 3 & 1.20 & 325.92 & 2.51 \\
\hline 2 & 1.70 & 488.89 & 2.69 \\
\hline 1 & 2.81 & 977.77 & 2.99 \\
\hline 0.5 & 4.40 & 1955.55 & 3.29 \\
\hline 0.25 & 6.63 & 3911.1 & 3.59 \\
\hline 0.142 & 9.11 & 6868.25 & 3.84 \\
\hline 0.0996 & 11.09 & 9820.49 & 3.99 \\
\hline
\end{tabular}
of the highest-redshift galaxy yet observed).

Table 1. Cosmic age, redshift $z$, Hubble parameter, $\log _{10}$ Hubble parameter. 
The results shown in Table 1 can be used to present a graph (Figure 1) of $\log _{10}\left(H_{t}\right)$ as a function of redshift $z$.

\section{Discussion}

The FSC publication entitled, "Temperature Scaling in Flat Space Cosmology in Comparison to Standard Cosmology" [11] makes note of the fact that the temperature curves of FSC and $\Lambda$ CDM cosmology are somewhat different, particularly in the first billion years or so of cosmic evolution. This is a function of the embedded FSC scaling thermodynamic equation. A graph (Figure 2) from the temperature scaling paper is reproduced herein.

The blue line is the radiation temperature $\left(T_{R}\right)$ curve expected in the $\Lambda$ CDM concordance model and the green line is the radiation temperature curve expected in FSC. The dashed red line represents the measured and projected spin temperature $\left(T_{S}\right)$ and the solid red line represents the baryonic gas temperature

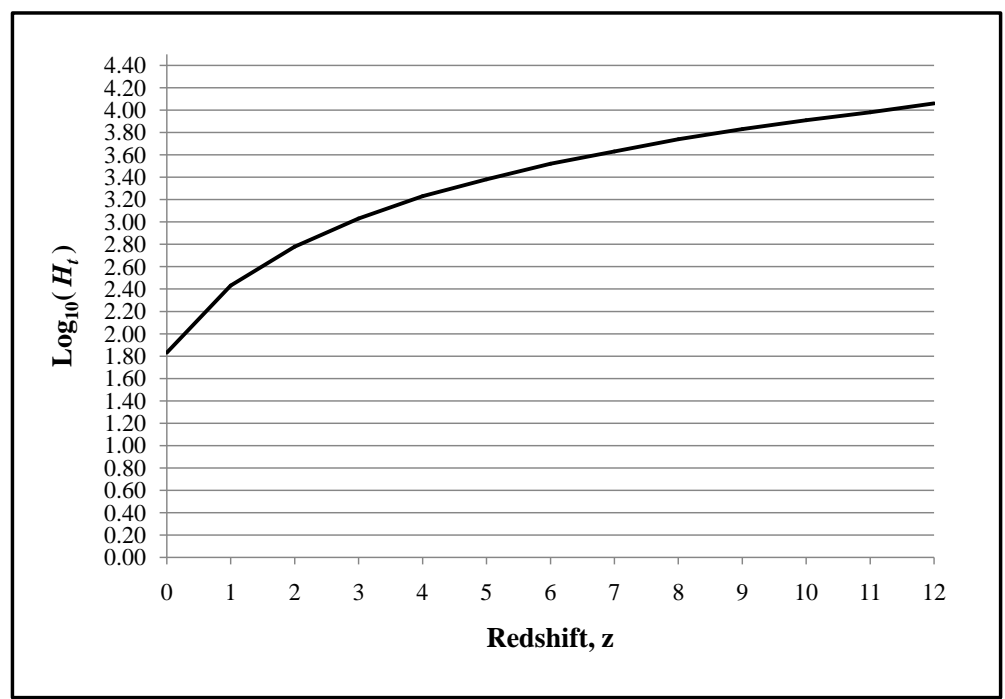

Figure $1 . \log _{10}\left(H_{t}\right)$ as a function of redshift $z$.

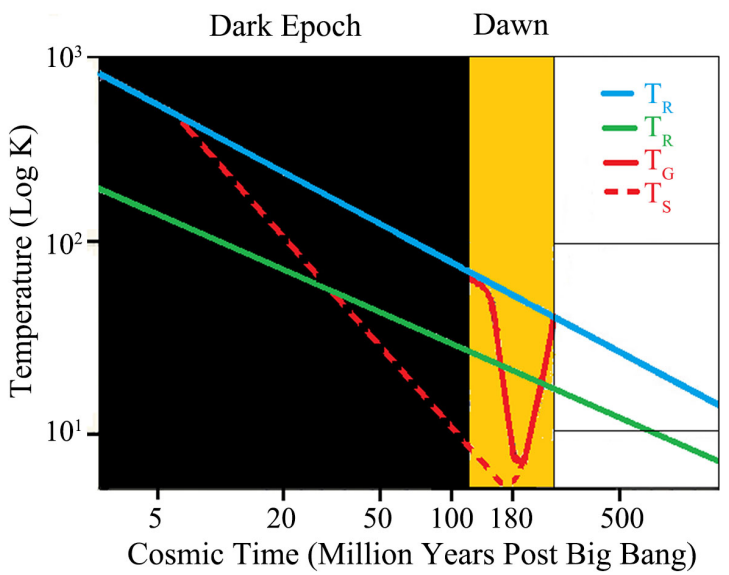

Figure 2. Cosmic temperature vs cosmic age in $\Lambda \mathrm{CDM}$ (blue) and FSC (red). 
$\left(T_{G}\right)$. In Figure 2, the concordance model temperature curve, as well as the concordance model conception of the time scale at which "cosmic dawn" occurred is presented as seen in Bowman's publication [12] on the recent redshifted $21-\mathrm{cm}$ observations. One should note that, in the FSC model, the same radiation temperatures and redshift $z$ values correlating to "cosmic dawn" occurred at about 20 - 60 million years after the Planck epoch, as opposed to approximately 110 - 250 million years in the concordance model. As discussed in recent FSC papers (see references [10] and [11]), the FSC model allows for earlier galaxy formation by several hundred million years in comparison to the concordance model. Thus, the FSC model, by its significantly colder temperature curve in the first billion years, offers a solution to the "The Impossibly Early Galaxy Problem" described by Steinhardt [13].

Of most importance with respect to the stated purpose of this paper, one can now see that the FSC model makes very specific predictions for the Hubble parameter values correlating with the range of currently observable galactic redshifts. The following major points need to be stressed:

1) As is true for all $R_{h}=c t$ models, the FSC Hubble parameter is scalar over the great span of cosmic time. $R_{h}=c t$ models require that the Hubble parameter value be defined according to $H_{t}=c / R_{t}$ where $c$ is the speed of light and $R_{t}$ is the Hubble radius at any time $t$. Thus, while at any point in human time spans the Hubble parameter may appear to be a constant, any cosmic model which incorporates $c / R_{t}$ within its Hubble parameter equation stipulates that the Hubble term is not a true constant over the great span of cosmic time.

2) The SHOES study of the "local" Hubble constant measured galactic separation velocities per megaparsec at earlier cosmic times corresponding to a range of redshift $z$ values of less than 0.15 . It is interesting to note that their averaged "outlier" Hubble constant value corresponds roughly with the FSC Hubble parameter value correlated to a redshift $z$ value of 0.06 (see Table 1). Moreover, when the Milky Way was used as a sole anchor for these observations, the SHOES study $H_{0}$ value of $76.18 \pm 2.37 \mathrm{~km} \cdot \mathrm{s}^{-1} \cdot \mathrm{Mpc}^{-1}$ can be seen to fit the middle of the $0<z<0.15$ range of our Figure 1 curve. The $\log _{10}(76.18)$ is 1.88 . This may be more readily apparent if one compares the first five entries in Table 1 . Similar adopted zero point measurements of Hubble parameters corresponding to higher $\mathrm{z}$ value ranges are fully expected by these authors to correlate with higher sections of Figure 1 curve. If so, then modern cosmologists will soon accept the idea of a time-variable, scalar, Hubble parameter.

3) Table 1 and Figure 1 show very specific predictions of the FSC model awaiting confirmation or refutation within the next few years of observations. If these observations prove that the Hubble parameter has a radically different temporal history than predicted herein, the FSC model and any similar $R_{h}=c t$ models would then be falsified. On the other hand, if observations in the next few years fit with Table 1 and Figure 1, the temporal behavior of dark energy would be better understood using the FSC model. In such case, the FSC model 
might then become the new concordance model.

\section{Summary and Conclusions}

The heuristic FSC $R_{h}=c t$ cosmology model is utilized to evaluate the tension between the SHOES local Hubble constant value and the Planck Survey and Dark Energy Survey global values. The SHOES report looked at a specific redshift $z$ range of $0<z<0.15$. Thus, the first approximately 11 billion years of the cosmic expansion history was not subjected to their analysis.

In the FSC model, the Hubble parameter is defined according to $H_{t}=c / R_{t}$ Therefore, the FSC Hubble parameter is time-variable and scalar according to Table 1 and Figure 1. The FSC model predicts that the middle of the range of 0 $<z<0.15$ should correlate with a Hubble parameter value of approximately 75 $\mathrm{km} \cdot \mathrm{s}^{-1} \cdot \mathrm{Mpc}^{-1}$, which fits the SHOES Hubble constant determination of $76.18 \pm$ 2.37 for the part of their study using the Milky Way as their anchor galaxy.

The authors predict that, in the next few years, similar measurements corresponding to higher $z$ value ranges will correlate with higher sections of Figure 1 curve. If this proves to be the case, then modern cosmologists will have to accept that what is currently called a Hubble constant is actually a time-variable, scalar, Hubble parameter. Furthermore, such a result would likely establish FSC as the new concordance model.

\section{Dedications and Acknowledgements}

This paper is dedicated to Dr. Stephen Hawking and Dr. Roger Penrose for their groundbreaking work on black holes and their possible application to cosmology. Dr. Tatum also thanks Dr. Rudolph Schild of the Harvard-Smithsonian Center for Astrophysics for his past support and encouragement. Author Seshavatharam UVS is indebted to professors Brahmashri M. Nagaphani Sarma, Chairman, Shri K.V. Krishna Murthy, founding Chairman, Institute of Scientific Research in Vedas (I-SERVE), Hyderabad, India, and to Shri K.V.R.S. Murthy, former scientist IICT (CSIR), Govt. of India, Director, Research and Development, I-SERVE, for their valuable guidance and great support in developing this subject.

\section{Conflicts of Interest}

The authors declare no conflicts of interest regarding the publication of this paper.

\section{References}

[1] Reiss, A.G., et al. (2016) The Astrophysical Journal, 826, 56. https://doi.org/10.3847/0004-637X/826/1/56

[2] Aghanim, N., et al. (2018) Planck 2018 Results VI. Cosmological Parameters.

[3] Macaulay, E., et al. (2018) Monthly Notices of the Royal Astronomical Society, 486, 2184-2196. 
[4] Melia, F. and Shevchuk, A.S. (2012) Monthly Notices of the Royal Astronomical Society, 419, 2579-2586. https://doi.org/10.1111/j.1365-2966.2011.19906.x

[5] Tatum, E.T., Seshavatharam, U.V.S. and Lakshminarayana, S. (2015) International Journal of Astronomy and Astrophysics, 5, 116-124.

https://doi.org/10.4236/ijaa.2015.52015

[6] Tatum, E.T., Seshavatharam, U.V.S. and Lakshminarayana, S. (2015) Journal of Applied Physical Science International, 4, 18-26.

[7] Tatum, E.T., Seshavatharam, U.V.S. and Lakshminarayana, S. (2015) Frontiers of Astronomy, Astrophysics and Cosmology, 1, 98-104.

[8] Tatum, E.T. and Seshavatharam, U.V.S. (2018) Journal of Modern Physics, 9, 2008-2020. https://doi.org/10.4236/jmp.2018.910126

[9] Tatum, E.T. and Seshavatharam, U.V.S. (2018) Journal of Modern Physics, 9, 1469-1483. https://doi.org/10.4236/jmp.2018.98091

[10] Tatum, E.T. (2018) Journal of Modern Physics, 9, 1867-1882. https://doi.org/10.4236/jmp.2018.910118

[11] Tatum, E.T. and Seshavatharam, U.V.S. (2018) Journal of Modern Physics, 9, 1404-1414. https://doi.org/10.4236/jmp.2018.97085

[12] Bowman, J.D. (2018) Nature, 555, 67-70. https://doi.org/10.1038/nature25792

[13] Steinhardt, C.L., et al. (2015) The Astrophysical Journal, 824, 21. https://doi.org/10.3847/0004-637X/824/1/21 